\title{
LncRNA BLACATI/miR-519d-3p/CREBI Axis Mediates Proliferation, Apoptosis, Migration, Invasion, and Drug-Resistance in Colorectal Cancer Progression
}

This article was published in the following Dove Press journal:

Cancer Management and Research

\author{
Rui Chen' \\ Shenkang Zhou' \\ Jianhui Chen' \\ Senbin Lin' \\ Feifei $\mathrm{Ye}^{\mathrm{I}}$ \\ Pinlu Jiang ${ }^{2}$
}

'Department of Gastroenterology, Taizhou Hospital of Zhejiang Province, Taizhou 317000, People's Republic of China; ${ }^{2}$ Department of Emergency, Taizhou Hospital of Zhejiang Province, Taizhou 317000, People's Republic of China
Correspondence: Pinlu Jiang Department of Emergency, Taizhou Hospital of Zhejiang Province, Linhai City, Taizhou City, Zhejiang Province, People's

Republic of China

Tel +86-1366577I795

Email ytfybr@163.com
Background: Colorectal cancer (CRC) is a common severe disease around the world. The merging papers reported that long noncoding RNAs (lncRNAs) took part in the diversified pathological processes of CRC. This study aimed to uncover the role and the potential mechanism of lncRNA bladder cancer-associated transcript 1 (BLACAT1) in CRC progression.

Methods: LncRNA BLACAT1, micro-519d-3p (miR-519d-3p), and cAMP-responsive element binding protein 1 (CREB1) levels were detected by quantitative real-time polymerase chain reaction (qRT-PCR) in CRC tissues and cells. The bio-functional effects were examined by 3-(4,5-dimethyl-2-thiazolyl)-2,5-diphenyl-2-H-tetrazolium bromide (MTT), flow cytometry assay, and transwell assay. The susceptibility testing was determined by oxaliplatin (OXA) administration. The potential binding sites between miR-519d-3p and BLACAT1 or CREBI were predicted by online software starBase and confirmed by dual-luciferase reporter analysis. The relative proteins expression in CRC cells was determined by Western blot analysis. Xenograft tumor model was used to evaluate biological function of BLACAT1 in vivo.

Results: The expression of BLACAT1 was promoted in CRC tissues and cells, and correlated to the TNM (tumor, node, metastasis) stage, distant metastasis, and overall survival rate. Silencing of BLACAT1 limited the proliferation, migration, and invasion, facilitated the apoptosis, and re-sensitized OXA-resistance in CRC cells. MiR-519d-3p was a target of BLACAT1. Furthermore, miR-519d-3p deletion reversed the positive effects of BLACAT1 deletion on CRC cells. Moreover, our data showed that miR-519d-3p directly targeted CREB1 and BLACAT1 sponged miR-519d-3p to regulate CREB1 expression. Besides, CREB1 disrupted the bio-functional results above from BLACAT1 suppression. Additionally, BLACAT1 knockdown promoted CRC cells sensitivity to OXA in vivo.

Conclusion: BLACAT1 mediated the progression of CRC and OXA-resistance by miR519d-3p/CREB1 axis.

Keywords: colorectal cancer, BLACAT1, oxaliplatin, miR-519d-3p, CREB1

\section{Highlights}

1. BLACAT1 is elevated in CRC tissues and cells.

2. BLACAT1 silencing suppresses CRC procession and oxaliplatin resistance.

3. BLACAT1 regulates $C R E B 1$ expression by sponging with miR-519d-3p.

4. BLACAT1 accelerates CRC progression via miR-519d-3p/CREB1 axis in vitro. 


\section{Introduction}

Colorectal cancer (CRC), an intractable malignancy, ranks the third leading cause in cancer-related death. ${ }^{1}$ Although the therapeutic method for CRC has achieved improvements, the overall survival rate was still low due to the high recurrence rate and drug resistance after chemotherapy treatment. ${ }^{2,3}$ Accumulating evidence indicated that $\mathrm{CRC}$ was considered as gene-related disease accompanied with distinctly pathological physiology and molecular features. Moreover, the dysfunction of long non-coding RNAs (lncRNAs) was found in CRC in recent years. ${ }^{4,5}$ Hence, a better understanding of the molecular mechanisms of CRC may provide the target for CRC treatment.

LncRNAs are a class of long RNAs (more than 200 nucleotides (nts) in length) without translation capacity and could affect gene expression at the transcriptional stage. ${ }^{6}$ Emerging evidence suggested that IncRNAs acted as tumor-suppressors or promoters, and widely participated in the processes of tumorigenesis. ${ }^{7,8}$ LncRNA bladder cancer-associated transcript 1 (lncRNA BLACAT1) was reported to be involved in various human cancers, such as glioma, ${ }^{9}$ papillary thyroid carcinoma, ${ }^{10}$ cervical cancer, ${ }^{11}$ colorectal cancer, ${ }^{12}$ and non-small cell lung cancer. ${ }^{13}$ Moreover, the high expression of lncRNA BLACAT1 in the serum of patients could work as a new supplementary biomarker for the diagnosis of CRC. ${ }^{14} \mathrm{Su}$ et al showed that BLACAT1 repressed p15 expression via binding with $E Z H 2$ and enhancing the regulation of cell cycle and proliferation in CRC cells. ${ }^{12}$ However, the role and underlying mechanism of BLACAT1 in CRC progression need further investigation.

MicroRNAs (miRNAs) are small non-protein-coding RNAs with 18 22 nucleotides, and they exhibit crucial effects on a series of cell biological behaviors, such as cell growth, survival, metastasis, and differentiation. ${ }^{15,16}$ Multiple researches indicated that the dysregulation of miRNAs was found in types of human diseases. ${ }^{17}$ MicroRNA-519d-3p (MiR-519d-3p) was confirmed to be dysregulated in some human cancers, such as non-small cell lung cancer, ${ }^{18}$ pancreatic ductal adenocarcinoma, ${ }^{19}$ and gastric cancer. ${ }^{20}$ Moreover, Ye et al reported that miR-519d-3p was downregulated in CRC tissues and cells. $^{21}$ However, the molecular mechanism of miR519d-3p in CRC progression remains largely unclear.

In this study, we aimed to explore the role and the underlying mechanism of lncRNA BLACAT1 in human
CRC, as well as investigating the effect of BLACAT1/ miR-519d-3p axis on the progression of CRC.

\section{Materials and Methods Clinical Data}

The experiment was authorized by the Ethics Committee of Taizhou Hospital of Zhejiang Province (201924-13) and executed according to the Declaration of Helsinki Principles. Paired colorectal cancer tumors and adjacent normal mucosa tissues were obtained from 55 CRC patients (23 males and 32 females; age: $56-70$ years old) were collected at the Department of General Surgery, Taizhou Hospital of Zhejiang Province between September 2012 and December 2014. CRC patients who received neoadjuvant chemotherapy/radiotherapy were excluded. The tumor stage was classified according to the 7th edition of the AJCC Cancer Staging Manual (2010) and CRC patients were divided into two groups: T1/T2 stage $(n=25)$ and T3/T4 stage $(n=30)$. Written informed consents were provided by all participants. All samples were preserved at $-80^{\circ} \mathrm{C}$ for storage.

\section{Cell Culture and Transfection}

Normal human intestinal epithelial cell-6 (HIEC-6) cell line, four diverse CRC cell lines (HCT116, HT29, LOVO, and SW620), and OXA-resistant cell lines (HT29/OXA and HCT116/OXA) were obtained from Cell Center of Shanghai Institutes for Biological Sciences (Shanghai, China). All cells were cultured in n RPMI-1640 medium (Hyclone, Biowest) supplemented with $10 \%$ fetal bovine serum (FBS) and $1 \%$ penicillin/ streptomycin (Solarbio, Beijing, China).

Small interference RNA (siRNA) targeting BLACAT1 (si-BLACAT1\#1, si-BLACAT1\#2, si-BLACAT1\#3), BLACAT1 overexpression plasmid (BLACAT1), CREB1 overexpression plasmid (CREB1), miR-519d-3p mimic (miR-519d-3p), miR-519d-3p inhibitor (anti-miR-519d$3 p$ ), and the controls (si-NC, vector, miR-NC, anti-NC) were all obtained from GenePharm (Shanghai, China). Lipofectamine 3000 (Invitrogen, Carlsbad, CA, USA) was used for transfection according to the manufacturer's instructions. The sequences were shown as follows:

si-BLACAT1-1\#, sequence, 5'-GAATTAGAAGCGAG GGGTT-3';

si-BLACAT1-2\#, sequence, 5'-GACAGGCCACATTT GCCTA-3'; 
si-BLACAT1-3\#, sequence, 5'-GAATTAGGAGGAAG CGGTT-3';

\section{RNA Isolation and Quantitative Real-Time Polymerase Chain Reaction (qRT-PCR)}

Total RNA from tissues and cells were extracted by using TRIzol reagent (Life Technologies Corporation, Carlsbad, CA, USA), and reverse-transcribed using All-in-One ${ }^{\mathrm{TM}}$ miRNA Prime Script ${ }^{\mathrm{TM}} \mathrm{RT}$ reagent kit (Takara, Shiga, Japan) and Script RT reagent Kit (Takara). QRT-PCR was performed using the qRT-PCR Detection Kit (GeneCopoeia, Inc., Rockville, MD, USA) and SYBR mix (Takara) on the 7500 Fast Real-Time PCR system (Thermo Fisher Scientific, Waltham, MA, USA). U6 or GAPDH was used as an internal reference gene. The relative expression levels of BLACAT1, miR-519d-3p, CREB1 were calculated by the $2^{-\triangle \Delta C t}$ method. The sequences of primers for miR-519d-3p and U6 were designed and obtained from Sangon Biotech (Shanghai, China), and the sequences of primers for BLACAT1, miR519d-3p, CREB1, U6, and GAPDH were listed as follows:

BLACAT1 forward (5'-TGACGTCTTACTACACCC ATCCT-3'),

BLACAT1 reverse (5'-CTGCCACCTATAGGAAATG CG-3'),

miR-519d-3p forward (5'-CAAAGTGCCTCCCTTT-3'), miR-519d-3p reverse (5'-CAGTGCGTGTCGTGGA GT-3'),

CREB1 forward (5'-CCAGCAGAGTGGAGATGCA G-3'),

CREB1 reverse (5'-GTTACGGTGGGAGCAGATGA $\left.\mathrm{T}-3^{\prime}\right)$,

U6 forward (5'-GCTTCGGCAGCACATATACTAAA AT-3'),

U6 reverse (5'-CGCTTCACGAATTTGCGTGTCA T-3'),

GAPDH forward (5'-GACTCATGACCACAGTCCAT GC-3'),

GAPDH reverse (5'-AGAGGCAGGGATGATGTTC TG-3').

\section{3-(4,5-Dimethyl-2-Thiazolyl)- 2,5-Diphenyl-2-H-Tetrazolium Bromide (MTT)}

Cells $\left(5 \times 10^{3} /\right.$ well $)$ were seeded into the 96 -well plate and cultured for $24 \mathrm{~h}, 48 \mathrm{~h}$, and $72 \mathrm{~h}$. Then, MTT reagent (Sigma, St Louis, MO, USA) was added to each 96-well plate and incubated for further $4 \mathrm{~h}$. Subsequently, cell supernatant was discarded, and $200 \mu \mathrm{L}$ of DMSO (Solarbio) was added to dissolve intracellular formazan crystals. Cell proliferation was determined at $450 \mathrm{~nm}$ using a microplate reader (Thermo Fisher Scientific).

\section{Flow Cytometry}

Cells were collected by digesting with pancreatin and centrifuging. Then, cells were washed by iced phosphatebuffered saline (PBS) and resuspended with $1 \times$ binding buffer. Next, Annexin V-fluorescein isothiocyanate propidium iodide (Annexin V-FITC/PI) kit (BD Pharmingen, San Diego, CA, USA) was conducted to steps subsequently following the manufacturer's instructions. Apoptotic cells were examined using flow cytometer (BD Biosciences, Franklin Lakes, NJ, USA), and then the apoptosis rate was calculated.

\section{Transwell Assay}

Cell migration was detected by transwell chamber (Corning Life Sciences, Corning, NY, USA) without matrigel matrix, while cell invasion experiment was conducted with matrigel matrix (Corning). The lower chamber was added with RPMI1640 medium with $10 \%$ FBS, while the transfected HCT116 and HT29 cells were injected into the upper one with $100 \mu \mathrm{L}$ of serum-free medium. Cells on the lower surface of chambers were fixed by methanol and then stained with $0.1 \%$ crystal violet. Finally, a microscope was used to quantify the migrated or invaded cells.

\section{OXA Sensitivity Assay}

The cell viability of HT29/OXA and HCT116/OXA cells was determined by MTT assay kit (Sigma). OXA sensitivity was detected using the IC50 value (half maximal inhibitory concentration).

\section{Dual-Luciferase Assay}

The putative binding sites of miR-519d-3p and BLACAT1 or CREB1 were predicted by starBase software online. The amplified wild-type and the mutant fragment of BLACAT1 and CREB1 3'UTR were inserted into pMIR-REPOR luciferase vector (OBio Biology, Shanghai, China) to construct luciferase reporters, named as WT-BLACAT1, MUTBLACAT1, WT-CREB1, and MUT-CREB1. Then, WT-BLACAT1, MUT-BLACAT1, WT-CREB1, or MUTCREB1 and miR-519d-3p mimic or miR-NC were cotransfected into HCT116 and HT29 cells. The luciferase 
activity was tested using Dual-Lucy Assay Kit (Promega, Madison, WI, USA).

\section{Western Blot}

RIPA buffer (Solarbio) was used to isolate total proteins in cells, and the proteins were quantified by a NanoDrop 3000 (Thermo Fisher Scientific). Sodium dodecyl sulfatepolyacrylamide gel electrophoresis (SDS-PAGE) was used to separate proteins, and then proteins were transferred onto polyvinylidene fluoride (PVDF) membranes. Then, the membranes were blocked in skim milk for $2 \mathrm{~h}$ at $37^{\circ} \mathrm{C}$ and then incubated with primary antibodies at $4^{\circ} \mathrm{C}$ overnight. Following 2-h incubation with secondary antibody [Goat Anti-Rabbit IgG H\&L (HRP) (1:1000; ab205718, Abcam, Cambridge, UK)], the chemiluminescence was performed by using an ECL detection kit (Beyotime, Shanghai, China). The primary antibodies were as follows: anti-Cleaved caspase-3 (Cleaved-cas3; 1:500; ab32042, Abcam), MMP-9 (1:1000; ab76003, Abcam), antiCREB1 (1:1500; 12208-1-AP, Proteintech Group, Inc, China), and anti-GAPDH (1:5000; ab37168, Abcam).

\section{Xenograft Tumor Model}

The animal experiments were approved by the Ethic Committee of Taizhou Hospital of Zhejiang Province (201924-13) and performed in accordance with the National Institutes of Health's Guide for the Care and Use of Laboratory Animals. BALB/c-nude mice (four-weekold) were purchased from Charles River Laboratories (Beijing, China). HT29/OXA cells $\left(2.0 \times 10^{7}\right)$ transfected with sh-BLACAT1 or sh-NC were subcutaneously injected into the dorsal flanks of each nude mouse. One week later, the mice were intraperitoneally administered with $3 \mathrm{mg} / \mathrm{kg}$ OXA every 5 days, and PBS was used as the control. ${ }^{22}$ All mice were divided into 4 groups ( $\mathrm{n}=6$ per group): sh-NC, sh-BLACAT1, sh-BLACAT1+PBS, sh-BLACAT1+OXA, sh-NC group was used as a control group. The tumor volume was measured once per week with a digital caliper. The tumor volume was calculated with the equation: Volume $=\left(\right.$ length $\times$ width $\left.^{2}\right) / 2$. After 5 weeks, the mice were euthanized via $\mathrm{CO} 2$ asphyxia method using the flow rate of $\mathrm{CO} 2$ to displace $50 \%$ air of cage volume per min in line with the current guideline of American Veterinary Medical Association (AVMA). Tumor tissues were separated for tumor weighing, qRT-PCR, and Western blot assay.

\section{Statistical Analysis}

All data were expressed as mean \pm standard deviation (SD) and analyzed by the SPSS 17.0 software. Comparisons among different groups were analyzed using paired Student's $t$-test and one-way analysis of variance (ANOVA) followed by Tukey's test. A $P$ value less than 0.05 was regarded as statistically significant.

\section{Results}

\section{LncRNA BLACATI Expression Was Upregulated in CRC Tissues and Cells, and Correlated with TNM Stage, Distant Metastasis, and Overall Survival Rate}

Firstly, we examined the BLACAT1 level in the CRC tumor tissues. The expression of BLACAT1 was higher in $\mathrm{CRC}$ tissues $(\mathrm{n}=55)$ relative to the adjacent normal tissues ( $\mathrm{n}=55)$ (Figure 1A). Next, we explored the correlation between BLACAT1 expression level and the clinicopathological parameters in CRC patients. The expression of BLACAT1 in T1/T2 stage patients $(\mathrm{n}=25)$ and T3/T4 stage patients $(n=30)$ was measured by qRT-PCR. The results demonstrated that the BLACAT1 level was remarkably correlated with TNM stage, and the advanced TNM stage was significantly positively related to the high BLACAT1 expression (Figure 1B). Next, we assessed the role of BLACAT1 in CRC patients without distant metastasis $(\mathrm{n}=37)$ and CRC patients with distant metastasis $(\mathrm{n}=18)$. The data showed that high BLACAT1 level was significantly correlated with distant CRC metastasis (nonmetastasis, $n=37$ versus metastasis, $n=18$ ) (Figure $1 C$ ). To further evaluate the value of BLACAT1 in the prognosis of patients with CRC, we used Kaplan-Meier survival analysis and Log-rank tests. We divided the samples into high (above the mean, $\mathrm{n}=28$ ) and low (below the mean, $\mathrm{n}=27$ ) BLACAT1 expression groups according to the mean value of the BLACAT1 level. As shown in Figure 1D, the high BLACAT1 level was associated with shorter overall survival. Subsequently, BLACAT1 levels in four CRC cell lines (HCT116, HT29, LOVO, and SW620) and the normal human intestinal epithelial cell-6 (HIEC-6) cell line were determined using qRT-PCR. We observed that BLACAT1 expression was higher in CRC cell lines than in HIEC-6 (Figure 1E). 
A

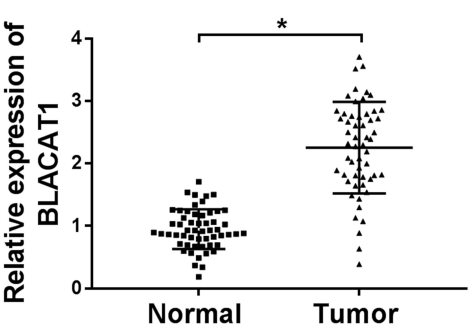

D

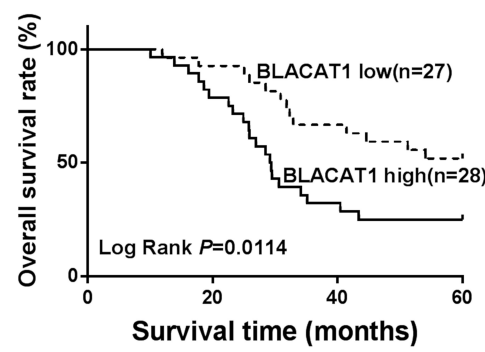

B

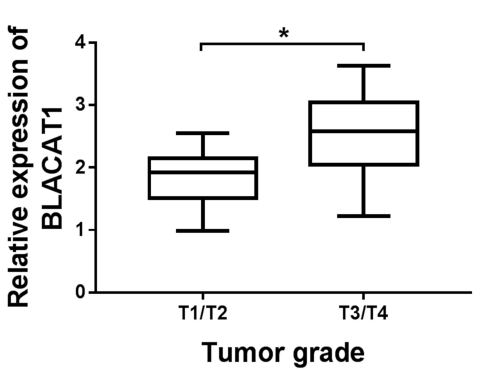

$\mathbf{E}$

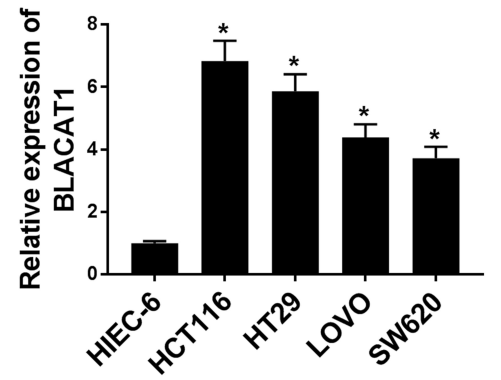

C

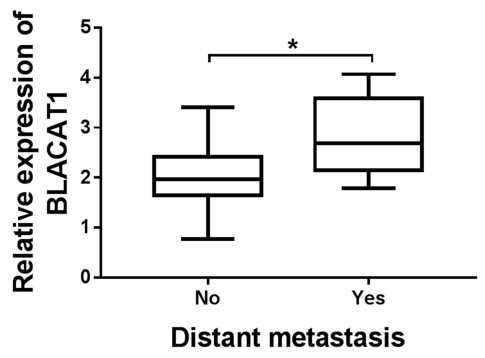

Figure I The expression of BLACATI in CRC tissues and cells, and its clinical parameters. (A) The expression level of BLACATI in CRC tissues and the corresponding normal tissues was detected by qRT-PCR. (B) A correlation analysis between the expression BLACATI and TNM stage was shown. (C) The relative expression of BLACATI in non-metastatic and metastatic human CRC tissues was determined by qRT-PCR. (D) The five-year survival rate of the patients with high and low expression of BLACATI was plotted by the Kaplan-Meier method. (E) The relative mRNA expression level of BLACATI was detected in cell lines (HIEC-6, HCTII6, HT29, LOVO, and SW620). $* P<0.05$

Abbreviations: BLACATI, bladder cancer-associated transcript I; CRC, colorectal cancer; TNM, tumor-node-metastasis.

\section{BLACATI Knockdown Suppressed the CRC Progression and Re-Sensitized OXA-Resistance of CRC Cells in vitro}

To further investigate the bio-functional effect of lncRNA BLACAT1 on CRC cells, we knocked down the BLACAT1 expression of HCT116 and HT29 cells using three different synthesized siRNA, si-BLACAT1\#1, si-BLACAT1\#2, si-BLACAT1\#3 individually. The expression level of BLACAT1 was downregulated by transfection of si-BLACAT1 (Figure 2A). Si-BLACAT1\#1 was used to conduct for further loss-functional experiments. MTT assay indicated that cell activity was significantly reduced by BLACAT1 deletion in HCT116 and HT29 cells (Figure 2B and C). Then, flow cytometry was used to detect the apoptosis, and the number of apoptotic cells was significantly increased in HCT116 and HT29 cells transfected with si-BLACAT1 (Figure 2D). Moreover, the abilities of migration and invasion were obviously suppressed by BLACAT1 knockdown (Figure 2E). Then, the expression of cell apoptosis-related protein Cleaved cas3 and cell metastasis-related protein MMP9 was detected by Western blot. Our data showed that BLACAT1 knockdown elevated Cleaved cas3 expression and suppressed MMP9 expression in HCT116 and HT29 cells (Figure 2F). MTT assay was performed to determine the IC50 of OXA in HT29/OXA and HCT116/OXA cells transfected with si-BLACAT1 or si-NC. Notably, the results showed that BLACAT1 knockdown resensitized HCT116/OXA and HT29/OXA cells to OXA (Figure $2 \mathrm{G}-\mathrm{J}$ ). These results indicated that knockdown of BLACAT1 repressed the abilities of proliferation, migration, and invasion, and promoted the rate of apoptosis cells, and re-sensitized CRC cells to OXA.

\section{MiR-519d-3p Was a Target miRNA of BLACATI}

Next, we predicted the relationship between BLACAT1 and miR-519d-3p by starBase, and the result showed that miR-519d-3p contained complementary sequences with BLACAT1 (Figure 3A). Then, we constructed dualluciferase reporter vectors and co-transfected miR-519d$3 p$ or miR-NC with BLACAT1-WT or BLACAT1-MUT into HCT116 and HT29 cells. Dual-luciferase reporter assays showed that miR-519d-3p reduced the luciferase activity of BLACAT1-WT, but not BLACAT1-MUT (Figure 3B and C). Furthermore, the expression level of miR-519d-3p was significantly upregulated by BLACAT1 

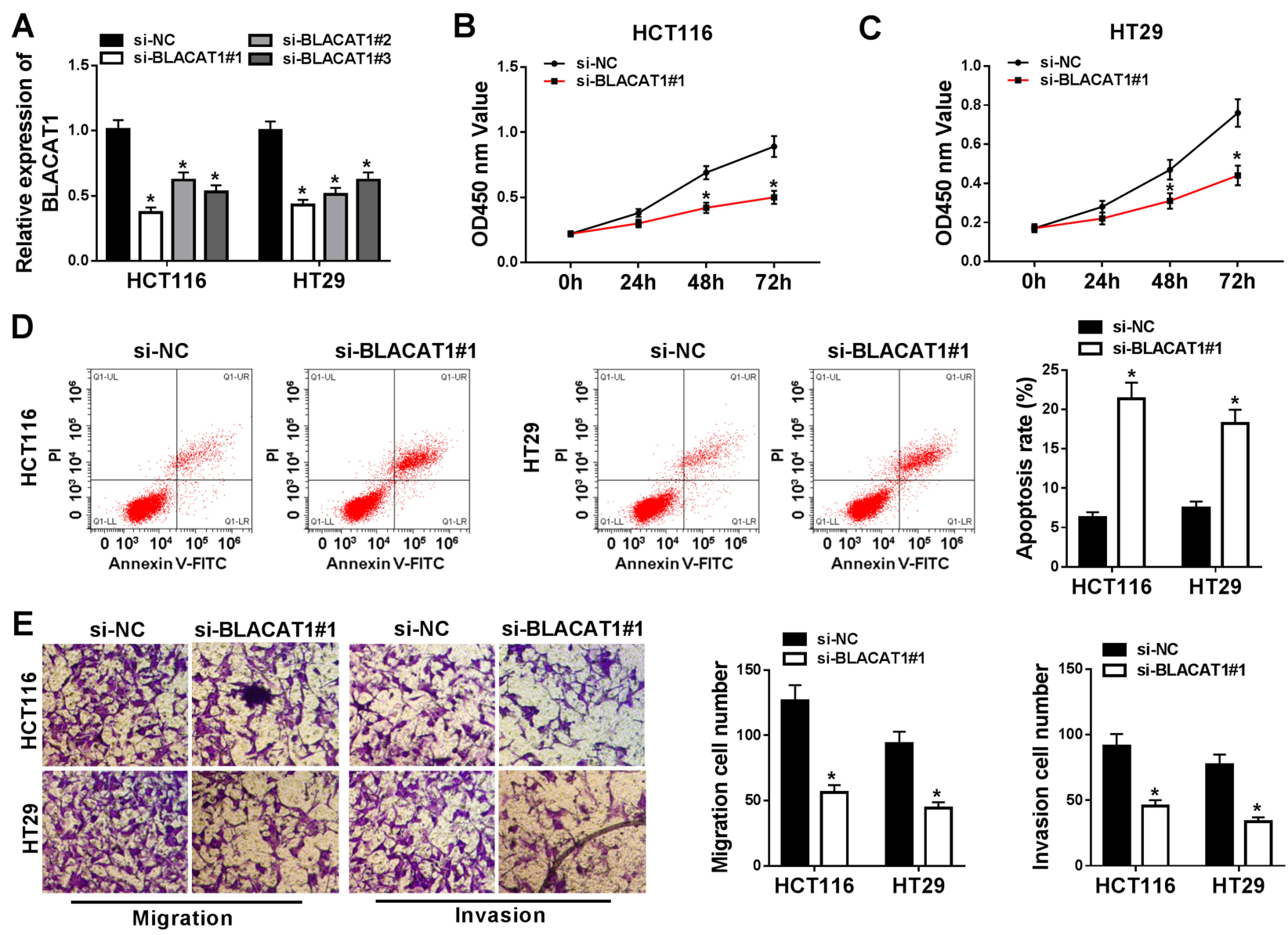

$\mathbf{F}$
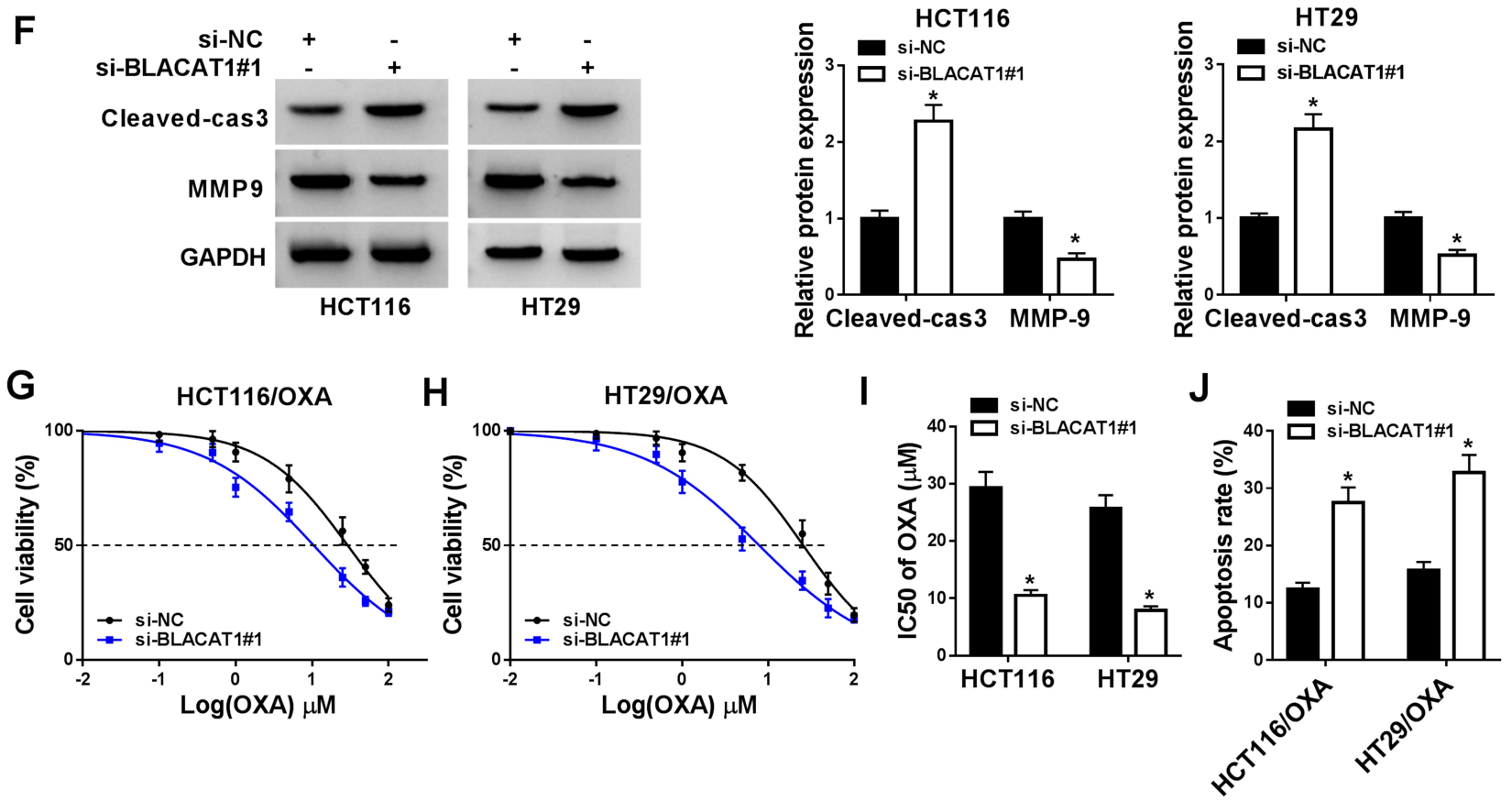

Figure 2 BLACATI regulated the bio-functional process of CRC and re-sensitized OXA-resistance in vitro. si-NC, si-BLACATI\#I, si-BLACATI\#2, and si-BLACATI\#3 were synthesized and transfected into CRC cells. (A) Identification of knockdown efficiency was analyzed by qRT-PCR. (B-C) The cell viability at determined times ( $24 \mathrm{~h}, 48$ h, $72 \mathrm{~h}$ ) was analyzed by MTT assay in HCTII6 and HT29 cells. (D) The apoptosis rate was confirmed by flow cytometry. (E) Cell migration and invasion were evaluated by transwell assay. (F) The protein expression of Cleaved-cas3 and MMP-9 was detected by Western blot. (G-I) MTT assay was performed to evaluate IC50 of OXA. (J) Cell apoptosis was determined by flow cytometry analysis in si-BLACATI\#I or si-NC transfected HCTII6/OXA and HT29/OXA cells after $20 \mu M$ OXA exposure. *P<0.05. Abbreviations: BLACATI, bladder cancer-associated transcript I; CRC, colorectal cancer; OXA, oxaliplatin; IC50, half-maximal inhibitory concentration. 
A

BLACAT1 WT 5' GCACCUGGagUAAGCACUUUU 3'

miR-519d-3p 3' GUGAGAUUUCCCUC || | | || |

BLACAT1 MUT 5' GCACCUGGAGUAAAUGACGCU 3'
B

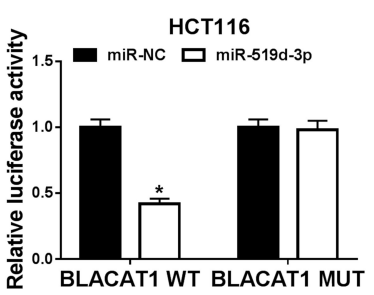

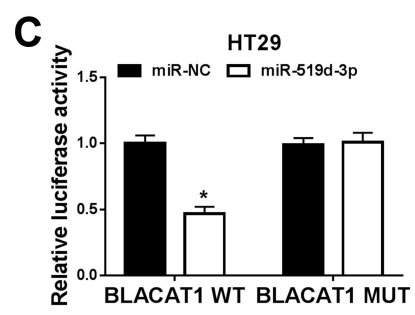

D

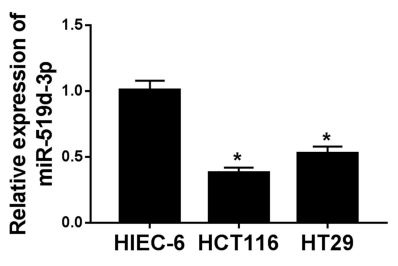

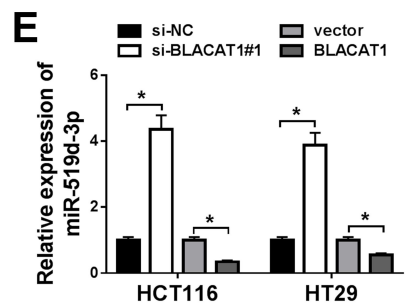

\section{$\mathbf{F}$}

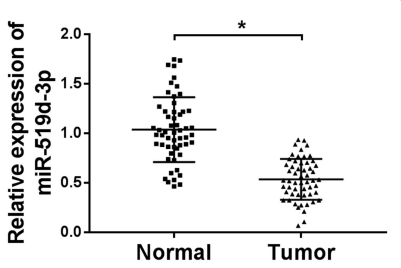

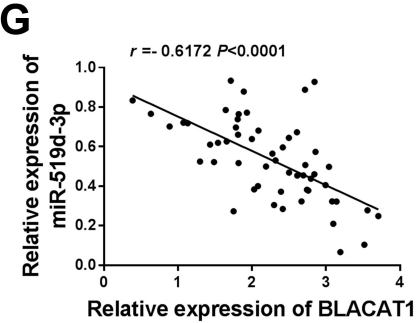

Figure 3 BLACATI was a direct target of miR-5I9d-3p. (A) The putative binding sites between miR-5I9d-3p and BLACATI were predicted by starBase. (B and C) The predicted sites were identified by dual-luciferase reporter assay. Vector and BLACATI were designed to explore the effects of miR-5I9d-3p by loss-and-gain functional experiment on CRC cells. (E) QRT-PCR was used to detect miR-5I9d-3p level in each group. (D and F) MiR-5I9d-3p expression in tissues and cells was evaluated by qRTPCR. (G) A correlation analysis between BLACATI and miR-5I9d-3p expression was performed. $* P<0.05$.

Abbreviations: BLACATI, bladder cancer-associated transcript I; miR-5I9d-3p, microRNA-5I9d-3p; CRC, colorectal cancer.

deletion and downregulated by BLACAT1 overexpression in HCT116 and HT29 cells (Figure 3E). Besides, we also evaluated that the expression patterns of miR-519d-3p in CRC cells and tissues of CRC patients, and the data represented that the level of miR-519d-3p was decreased in both CRC cells and tissues, compared with its own corresponding controls (Figure 3D and F). In addition, the expression relationship between miR-519d-3p and BLACAT1 kept a negative correlation (Figure 3G). Therefore, we determined that miR-519d-3p was the target miRNA of BLACAT1.

\section{MiR-519d-3p Silencing Reversed the Effect of BLACATI Knockdown on CRC Cells in vitro}

To better explore the functional relationship between BLACAT1 and miR-519d-3p, HCT116 and HT29 cells were transfected with miR-NC, miR-519d-3p, siBLACAT1\#1+anti-miR-NC, or si-BLACAT1\#1+anti-miR $-519 d-3 p$. Firstly, the low expression of miR-519d-3p was confirmed by qRT-PCR analysis in the si-BLACAT1\#1 +anti-miR-519d-3p group (Figure 4A). Thereafter, a significant decrease of cell viability and the increase of cell apoptosis were induced by miR-519d-3p overexpression or BLACAT1 deletion, while the effects of BLACAT1 deletion on cell viability and apoptosis were reversed by miR-519d-3p inhibitor (Figure 4B-D).
Meanwhile, cell migration and invasion were suppressed by miR-519d-3p overexpression or BLACAT1 deletion, while the effect of BLACAT1 deletion on cell migration and invasion was blocked by miR-519d-3p inhibitor (Figure 4E and $\mathrm{F}$ ). Western blot results indicated that miR-519d-3p overexpression or BLACAT1 knockdown promoted the expression of Cleaved-cas3 and inhibited the expression of MMP-9, while these effects of BLACAT1 knockdown were reversed by miR-519d-3p inhibitor (Figure 4G-I). Moreover, BLACAT1 deletion promoted the sensitivity of HCT116 and HT29 cells to OXA, which was abolished by miR-519d-3p inhibitor (Figure 4J-K). These results indicated that miR-519d-3p inhibitor inverted the effects of si-BLACAT1 transfection, and suppressed the sensitized OXA-resistance in CRC cells.

\section{CREBI is a Target Gene of miR-5I9d-3p}

The starBase prediction showed that $C R E B 1$ was a potential target of miR-519d-3p (Figure 5A). Then, we co-transfected miR-519d-3p or miR-NC with CREB1-WT or CREB1-MUT into HCT116 and HT29 cells. Dualluciferase reporter assay showed that overexpression of miR-519d-3p reduced the luciferase activity of CREB1WT, but not CREB1-MUT (Figure 5B and C). Besides, overexpression of miR-519d-3p might significantly decrease $C R E B 1$ expression, while anti-miR-519d-3p significantly increased CREB1 expression in HCT116 and 

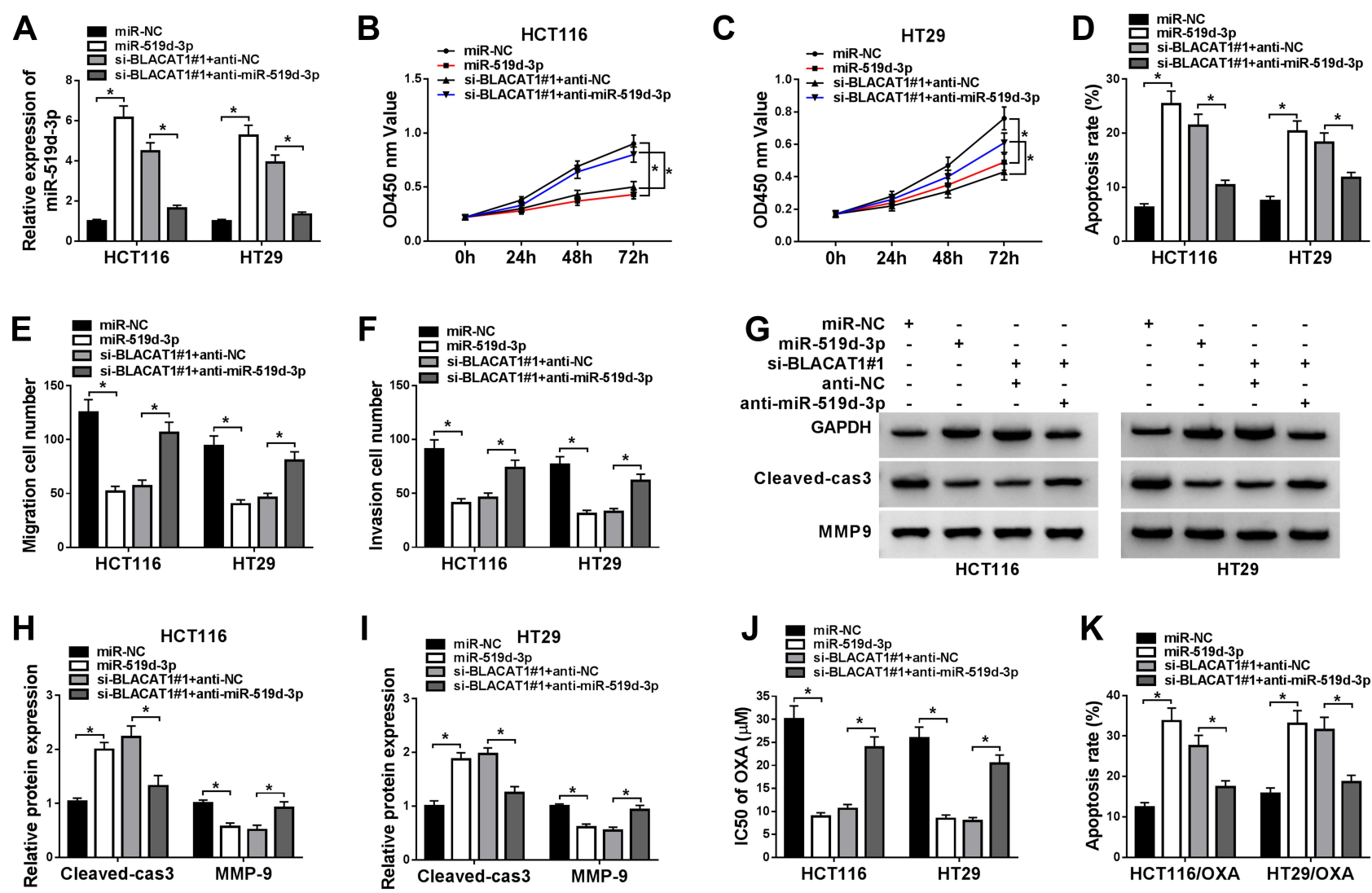

Figure 4 Knockdown of miR-5I9d-3p inverted the functional effects of BLACATI deletion, and decreased the OXA sensitivity of CRC cells in vitro. MiR-NC, miR-5 I9d-3p, si-BLACATI\#I+anti-NC or si-BLACATI\#I+anti-miR-5I9d-3p was separately transfected into CRC cells. (A) QRT-PCR was used to confirm the level of miR-5I9d-3p in each group. (B-C) The cell viability was analyzed by MTT assay at stated times ( 24 h, 48 h, 72 h) in HCTII 6 and HT29 cells. (D) Flow cytometry was conducted to analyze the cell apoptosis rate. (E-F) Cell migration and invasion were evaluated by transwell assay. (G-I) The expression of Cleaved-cas 3 and MMP-9 was measured by Western blot. HCTI I6/OXA and HT29/OXA cells were given similar administration above, (J) the IC50 of OXA was determined by MTT assay, and (K) the cell apoptotic rate was explored by flow cytometry analysis. $* P<0.05$.

Abbreviations: BLACATI, bladder cancer-associated transcript I; miR-5I9d-3p, microRNA-5I9d-3p; CRC, colorectal cancer; OXA, oxaliplatin; IC50, half maximal inhibitory concentration.

HT29 cells (Figure 5D and E). Furthermore, BLACAT1 deletion suppressed CREB1 expression, which was reversed by miR-519d-3p inhibitor (Figure 5F-G).

\section{Replenishment of CREBI Reversed the Effect of BLACATI Downregulation on CRC Cells}

To explore the functional relationship between BLACAT1 and $C R E B 1$, the si-NC, si-BLACAT1\#1, si-BLACAT1\#1 +vector or si-BLACAT1\#1+CREB1 was transfected into HCT116 and HT29 cells, individually. Firstly, we examined the expression of CREBI in CRC cells by Western blot and found that the level of CREBI was significantly inhibited by BLACAT1 knockdown, which was abolished by CREB 1 overexpression (Figure 6A). Then, the promotion effect of BLACAT1 knockdown on cell apoptosis, and the inhibition effects on cell viability, migration, and invasion were reversed by CREB1 overexpression (Figure 6B-F). Our data showed that the promotion effect of BLACAT1 knockdown on Cleaved-cas3 expression, and the inhibition effect on MMP-9 expression were abolished by CREB1 overexpression (Figure 6G and $\mathrm{H}$ ). Also, the sensitivity of HCT116/OXA and HT29/OXA cells was arrested when cells were transfected with si-BLACAT1\#1 $+C R E B 1$ (Figure 6I-J). The results indicated that overexpression of CREB1 reversed the effects of BLACAT1 downregulation on CRC cells in vitro.

\section{BLACATI Knockdown Enhanced OXA-Sensitivity in CRC in vivo}

To further determine the functional role of BLACAT1 in OXA resistance in vivo, HT29/OXA cells infected with shNC or sh-BLACAT1 were subcutaneously injected into the nude mice to generate xenograft, followed by treatment with OXA or PBS. The result indicated that BLACAT1 
A

$\begin{aligned} \text { CREB1 WT 3'UTR 5' GUUUGGUGUAACUUGCACUUUU 3' } & \\ \text { miR-519d-3p } & \text { 3' GUGAGAUUUCCCUCCGUGAAAC 5' }\end{aligned}$

CREB1 MUT 3'UTR 5' GUUUGgUGUAACUUAUGACGCU 3'
B

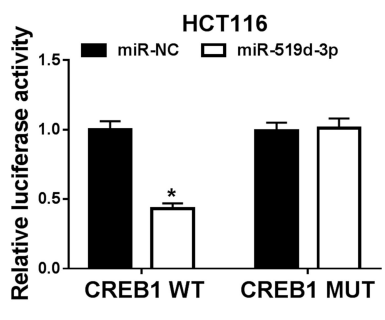

D

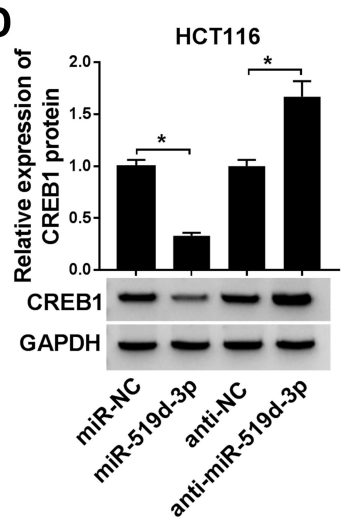

E

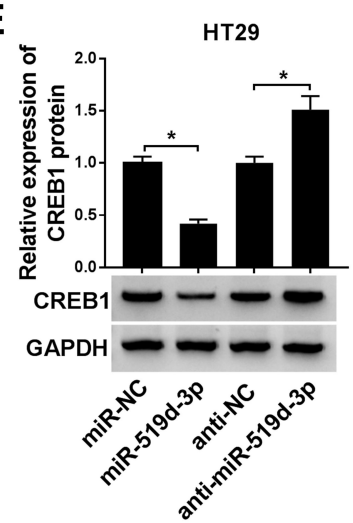

$\mathbf{F}$
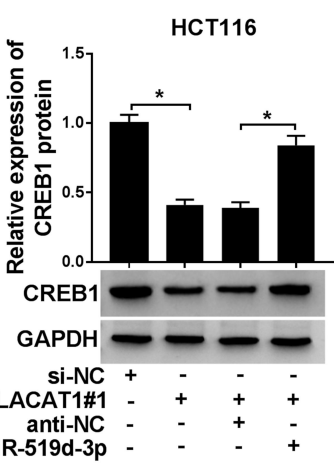

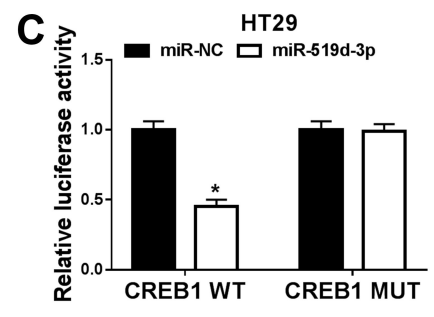

G

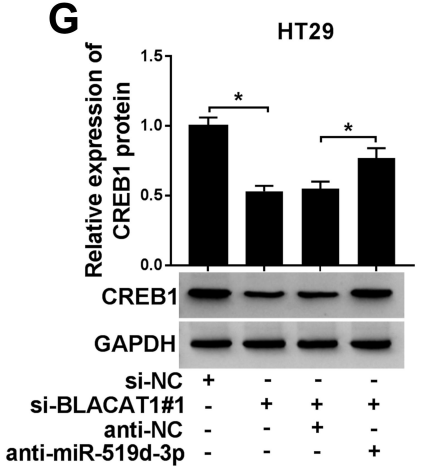

Figure 5 CREBI was a target gene of miR-5I9d-3p. (A) CREBI was predicted by starBase as a potential target for miR-5I9d-3p. (B and C) Dual-luciferase reporter assay was conducted to verify the interaction between miR-5I9d-3p and CREBI. (D and E) The expression of CREBI after loss-and-gain of the miR-5I9d-3p experiment was measured in HCTII 6 and HT29 cells using Western blot. (F and $\mathbf{G})$ The expression of CREBI after transfection with si-BLACATI\#I or anti-miR-5I9d-3p was detected in HCTII 6 and HT29 cells using Western blot. $* P<0.05$.

Abbreviations: CREBI, cAMP responsive element binding protein I; miR-519d-3p, microRNA-5I9d-3p.

knockdown or OXA treatment significantly suppressed tumor growth, evidenced by the reduced tumor volume and tumor weight. Moreover, simultaneous BLACAT1 knockdown and OXA treatment resulted in a more obvious inhibition on tumor growth, suggesting that down-regulation of BLACAT1 facilitated the sensitivity of CRC cells to OXA in vivo (Figure 7A and B). Furthermore, qRT-PCR analysis showed that a lower mRNA level of BLACAT1 and a higher expression of miR-519d-3p was found in tumors after shBLACAT1 introduction (Figure 7C and D), especially after the combination of sh-BLACAT1 introduction and OXA treatment. Western blot assay revealed that BLACAT1 knockdown greatly weakened CREB1 and MMP-9 protein level, and promoted Cleaved-cas3 expression in tumor tissues (Figure 7E). The combination of BLACAT1 knockdown and OXA exposure led to much lower CREB1 and MMP-9 protein expression and higher Cleaved-cas 3 expression (Figure 7E). All these data demonstrated that BLACAT1 depletion enhanced the OXA sensitivity of CRC cells in vivo.

\section{Discussion}

LncRNAs have been reported to be involved in the progression of colorectal cancer (CRC). In recent three years, several novel IncRNAs with abnormal expression have been defined in CRC $^{23-27}$ Zhong et al found that MLK7 antisense RNA 1
(MLK7-AS1) was significantly upregulated in both CRC tissues and lines. Meanwhile, the increased MLK7-AS1 expression was positively correlated to a series of clinicopathological factors in patients with $\mathrm{CRC} .{ }^{28} \mathrm{Li}$ et al reported that long noncoding RNA FGD5 antisense RNA 1 (FGD5-AS1) was increased in CRC cells9. ${ }^{29}$ However, the molecular mechanisms of lncRNAs in CRC progression have not been fully understood yet.

Several studies showed that BLACAT1 participated in multiple cancers. High expression of lncRNA BLACAT1 was considered to be a new prognostic predictor of tumors. ${ }^{14}$ For instance, the enhanced expression of BLACAT1 correlated with advanced stage, metastasis, and poor prognosis of participants with non-small cell lung cancer (NSCLC). ${ }^{14,30}$ Previous studies documented that BLACAT1 exerted vital patterns in the progression of CRC. Enhanced expression of BLACAT1 was an independently poor prognostic indicator for $\mathrm{CRC}$, and downregulation of BLACAT1 significantly inhibited tumor proliferation and invasion in vitro. ${ }^{12,31}$ Furthermore, high expression of BLACAT1 contributed to resistance to tumor cells with OXA or cisplatin treatment, which suggested that BLACAT1 may participate in tumor progression and chemical resistance. ${ }^{9,32}$

Overexpression of miR-519d-3p in vitro could significantly inhibit the abilities of proliferation, migration, and 

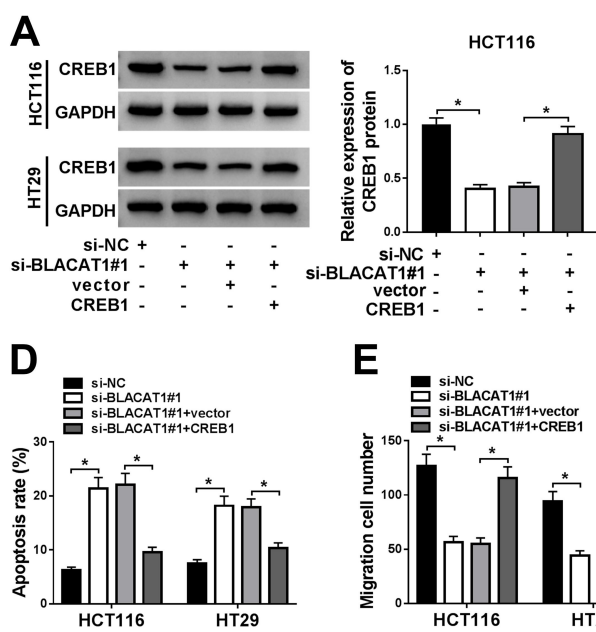

E
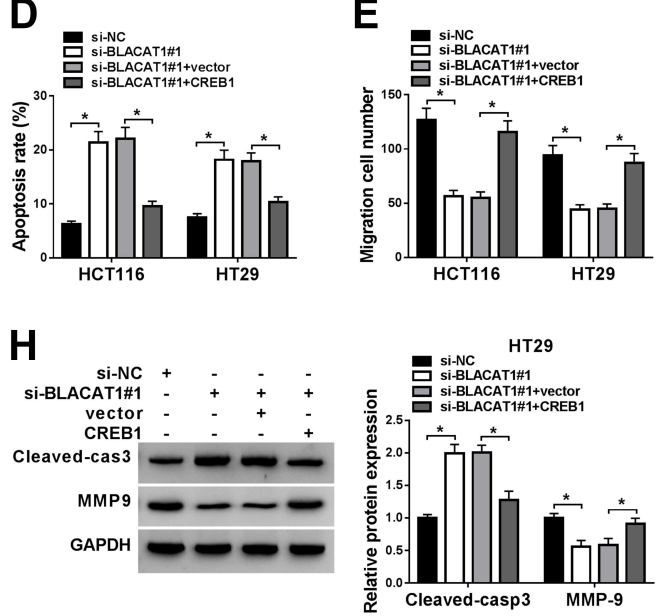
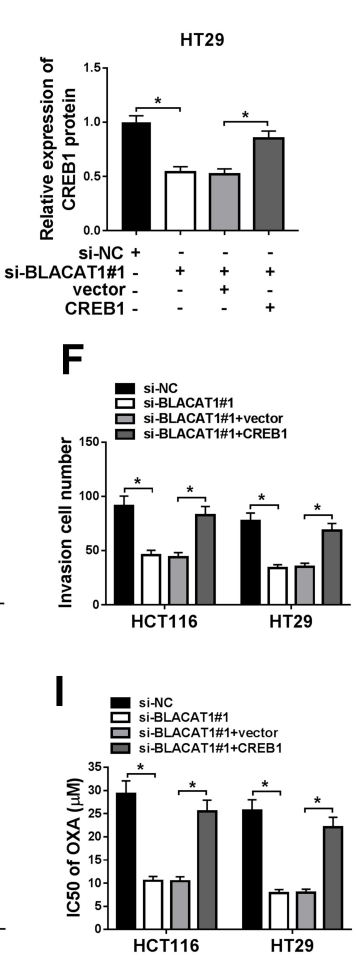
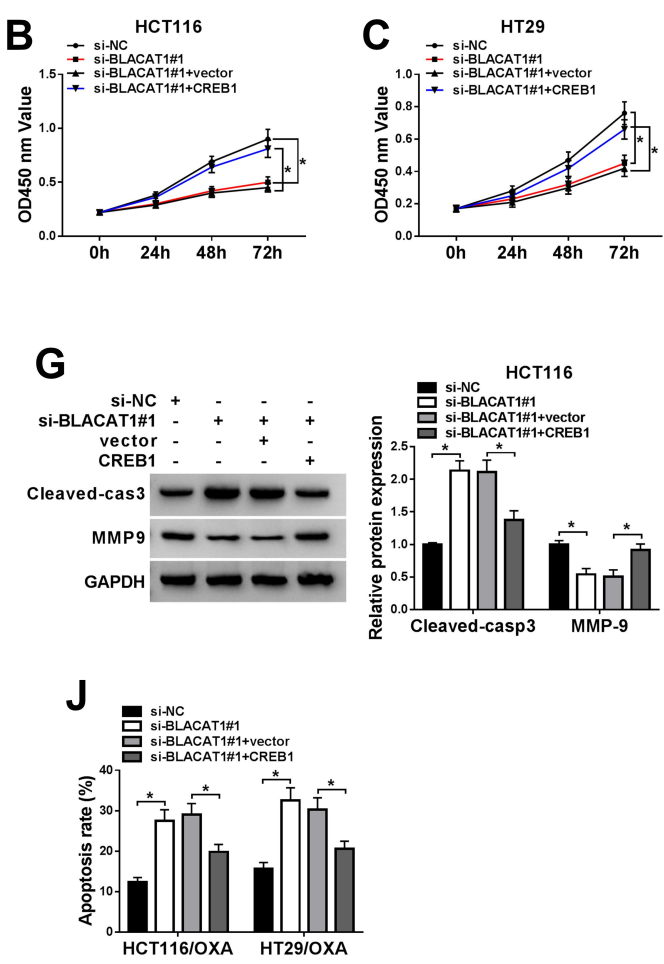

Figure 6 Overexpression of CREBI reversed the functional effects of BLACATI deletion on CRC progression, and limited the OXA sensitivity of CRC cells in vitro. Si-NC, si-BLACAT\#I, si-BLACATI\#I+vector or si-BLACATI\#I+CREBI was separately transfected into CRC cells. (A) Western blot was used to confirm the level of CREB I of two different CRC cells in each group. (B and C) Cell viability was analyzed by MTT assay at determined times (24 h, 48 h, 72 h) in HCTI I6 and HT29 cells. (D) Flow cytometry was conducted to analyze the cell apoptosis rate. (E and $\mathbf{F})$ Cell migration and invasion were evaluated by transwell assay. (G and $\mathbf{H})$ The expression of Cleaved-cas 3 and MMP-9 was determined by Western blot. The equal transfected treatment, as shown above, was carried out into HCTII6/OXA and HT29/OXA cells, (I) the IC50 of OXA was determined by MTT assay, and (J) the cell apoptotic rate was explored by flow cytometry analysis. $* P<0.05$.

Abbreviations: CREBI, cAMP responsive element binding protein I; BLACATI, bladder cancer-associated transcript I; miR-5I9d-3p, microRNA-5I9d-3p; CRC, colorectal cancer; OXA, oxaliplatin; IC50, half maximal inhibitory concentration.

invasion in CRC cells, as well as increase the sensitivity of cells to 5-fluorouracil (5-Fu) exposure. ${ }^{33-35}$ Our data showed that the high expression of BLACT1 positively correlated to TNM grade, distant metastasis, and overall survival, which was consistent with reported paper. ${ }^{12}$ Moreover, BLACAT1 knockdown suppressed cell viability, migration, invasion, and promoted cell apoptosis. Meanwhile, identical results also could be seen in osteosarcoma. $^{36}$ Furthermore, downregulated BLACT1 $^{3}$ re-sensitized OXA-resistance in vitro. Moreover, miR519d-3p was a potential target of BLACAT1. Meanwhile, function assays revealed that miR-519d-3p knockdown reversed the regulatory effects of BLACAT1 silencing on CRC cells. These results above showed that BLACAT1 could play an essential role in proliferation, apoptosis, migration, invasion, and drug-resistance in CRC progression in vitro by sponging miR-519d-3p.

$C R E B 1$ is an oncogene that regulates various processes including cell proliferation and differentiation. Studies have shown that inhibition of $C R E B 1$ expression could reduce the resistance of colorectal cancer cells to chemical drugs (OXA, 5-Fu). ${ }^{37,38}$ In our study, we identified that $C R E B 1$ was upregulated in CRC tissues and cells, and the similar results upon $C R E B 1$ in CRC were documented in the previous study. ${ }^{39}$ Interestingly, $C R E B 1$ was a target of miR-519d-3p. Moreover, the $C R E B 1$ protein expression was upregulated in $\mathrm{CRC}$ cells treated with co-transfection of both si-BLACAT1 and anti-miR-519d-3p in comparison with transfection of si-BLACAT1 solely in vitro. Function assays and drug-sensitivity tests demonstrated that the biological function regulated by BLACAT1 silencing could be inverted by $C R E B 1$ ectopic expression. Finally, diminished BLACAT1 facilitated the OXA-sensitivity of CRC cells in xenograft model experiments.

There were some limitations in this study, the interaction between miR-519d-3p and BLACAT1 or CREB1 was initially detected by dual-luciferase reporter assay, and it should be confirmed by RNA immunoprecipitation or RNA pull-down. Besides, the results and conclusions obtained using commercial cell lines could not fully represent the actual situation in vivo. 
A

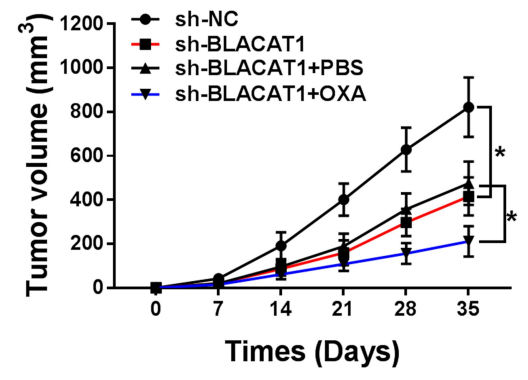

D

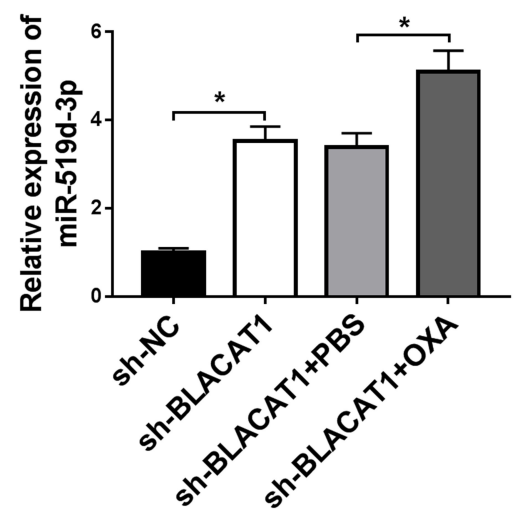

B

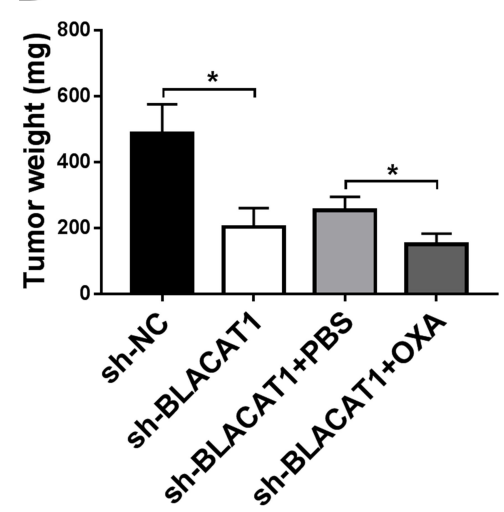

C

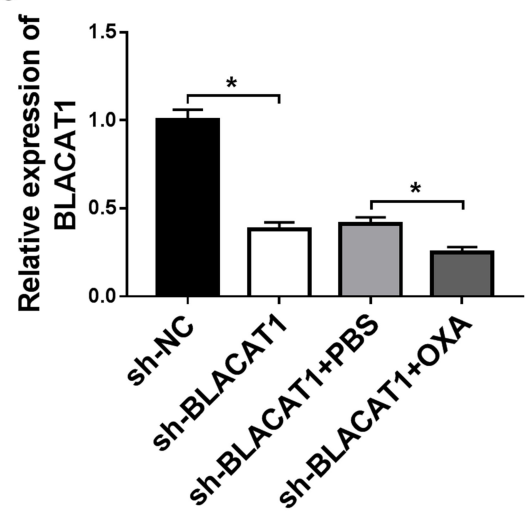

E

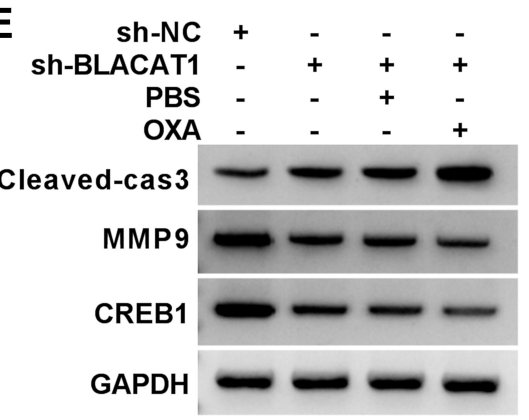

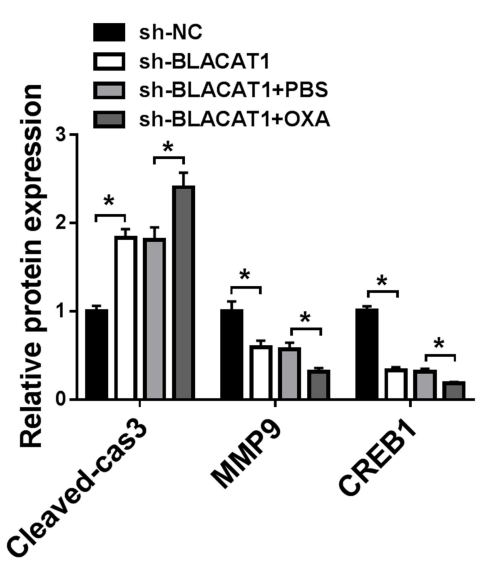

Figure 7 BLACATI knockdown sensitized CRC cells to OXA in vivo. HT29/OXA cells stably transfected with sh-NC or sh-BLACATI were subcutaneously inoculated into the nude mice, followed by treatment with PBS or OXA. Mice were euthanized to remove tumor masses at 35 days after inoculation. (A) The tumor volumes were measured with a caliper at indicated time points. (B) The average weight of resected tumors was detected. (C and D) QRT-PCR analysis was performed to measure BLACATI and miR-5 I9d-3p expression levels in excised tumor tissues. (E) The level of $C R E B I$, Cleaved-cas3 and MMP-9 was detected by Western blot analysis in excised tumor tissues. $* P<0.05$.

Abbreviations: BLACATI, bladder cancer-associated transcript I; CRC, colorectal cancer; OXA, oxaliplatin; miR-5I9d-3p, microRNA-5I9d-3p; CREBI, cAMP responsive element binding protein I.

In conclusion, our study uncovered that BLACAT1 worked as an oncogene, suppressed cell apoptosis, and OXA-induced sensitivity, facilitated cell proliferation, migration, and invasion by targeting miR-519d-3p to regulate CREB1 expression in CRC progression in vitro. Additionally, this paper also revealed a novel axis of miR-519d-3p/CREB1, supplying a different therapeutic method for CRC treatment.

\section{Funding}

This work was approved by Basic public welfare research plan of Zhejiang Province (LGF19H160017).

\section{Disclosure}

The authors declare that they have no financial or nonfinancial conflicts of interest.

\section{References}

1. Siegel R, Ma J, Zou Z, et al. Cancer statistics, 2014. CA Cancer J Clin. 2014;64(1):9-29. doi:10.3322/caac.21208

2. Centelles JJ. General aspects of colorectal cancer. ISRN Oncol. 2012;2012:139268.

3. Sunkara V, Hebert JR. The colorectal cancer mortality-to-incidence ratio as an indicator of global cancer screening and care. Cancer. 2015;121(10):1563-1569. doi:10.1002/cncr.29228

4. Saus E, Brunet-Vega A, Iraola-Guzman S, et al. Long Non-Coding RNAs As Potential Novel Prognostic Biomarkers in Colorectal Cancer. Front Genet. 2016;7:54.

5. Ding J, Lu B, Wang J, et al. Long non-coding RNA Loc554202 induces apoptosis in colorectal cancer cells via the caspase cleavage cascades. J Exp Clin Cancer Res. 2015;34:100.

6. Mercer TR, Dinger ME, Mattick JS. Long non-coding RNAs: insights into functions. Nat Rev Genet. 2009;10(3):155-159.

7. Fatica A, Bozzoni I. Long non-coding RNAs: new players in cell differentiation and development. Nat Rev Genet. 2014;15 (1):7-21.

8. Gibb EA, Brown CJ, Lam WL. The functional role of long non-coding RNA in human carcinomas. Mol Cancer. 2011;10:38. 
9. Wu X, Zheng Y, Han B, et al. Long noncoding RNA BLACAT1 modulates $\mathrm{ABCB} 1$ to promote oxaliplatin resistance of gastric cancer via sponging miR-361. Biomed Pharmacother. 2018;99:832-838.

10. Liao D, Lv G, Wang T, et al. Prognostic value of long non-coding RNA BLACAT1 in patients with papillary thyroid carcinoma. Cancer Cell Int. 2018;18(1):47. doi:10.1186/s12935-018-0544-9

11. Wang CH, Li YH, Tian HL, et al. Long non-coding RNA BLACAT1 promotes cell proliferation, migration and invasion in cervical cancer through activation of Wnt/beta-catenin signaling pathway. Eur Rev Med Pharmacol Sci. 2018;22(10):3002-3009.

12. Su J, Zhang E, Han L, et al. Long noncoding RNA BLACAT1 indicates a poor prognosis of colorectal cancer and affects cell proliferation by epigenetically silencing of p15. Cell Death Dis. 2017;8 (3):e2665. doi:10.1038/cddis.2017.83

13. Ye JR, Liu L, Zheng F. Long Noncoding RNA Bladder Cancer Associated Transcript 1 Promotes the Proliferation, Migration, and Invasion of Nonsmall Cell Lung Cancer Through Sponging miR-144. DNA Cell Biol. 2017;36(10):845-852. doi:10.1089/dna.2017.3854

14. Lu H, Liu H, Yang X, et al. LncRNA BLACAT1 May Serve as a Prognostic Predictor in Cancer: evidence from a Meta-Analysis. Biomed Res Int. 2019;2019:1275491. doi:10.1155/2019/1275491

15. Chen CZ, Li L, Lodish HF, et al. MicroRNAs modulate hematopoietic lineage differentiation. Science. 2004;303(5654):83-86. doi:10.1126/science. 1091903

16. Brennecke J, Hipfner DR, Stark A, et al. bantam encodes a developmentally regulated microRNA that controls cell proliferation and regulates the proapoptotic gene hid in Drosophila. Cell. 2003;113(1):25-36. doi:10.1016/S0092-8674(03)00231-9

17. Srinivasan S, Selvan ST, Archunan G, et al. MicroRNAs -the next generation therapeutic targets in human diseases. Theranostics. 2013;3(12):930-942. doi:10.7150/thno.7026

18. Ye Y, Zhao L, Li Q, et al. circ_0007385 served as competing endogenous RNA for miR-519d-3p to suppress malignant behaviors and cisplatin resistance of non-small cell lung cancer cells. Thorac Cancer. 2020;11(8):2196-2208. doi:10.1111/1759-7714.13527

19. Sun J, Zhang P, Tao Y, et al. Upregulation of LncRNA PVT1 Facilitates Pancreatic Ductal Adenocarcinoma Cell Progression and Glycolysis by Regulating MiR-519d-3p and HIF-1A. J Cancer. 2020;11(9):2572-2579. doi:10.7150/jca.37959

20. Jin W, Zhang H, Li M, et al. miR-519d-3p/HMGA2Long Noncoding RNA Regulator of Reprogramming Regulates Cell Growth, Metastasis, and Cisplatin Resistance in Gastric Cancer via Axis. Cancer Biother Radiopharm. 2020. doi:10.1089/cbr.2019.3525

21. Ye X, Lv H. MicroRNA-519d-3p inhibits cell proliferation and migration by targeting TROAP in colorectal cancer. Biomed Pharmacother. 2018;105:879-886.

22. Li T, Jin X, Dong J, et al. Long noncoding RNA ARSR is associated with a poor prognosis in patients with colorectal cancer. $J$ Gene Med. 2020; 3241.

23. Tang J, Yan T, Bao Y, et al. LncRNA GLCC1 promotes colorectal carcinogenesis and glucose metabolism by stabilizing c-Myc. Nat Commun. 2019;10(1):3499. doi:10.1038/s41467-019-11447-8

24. Wu Y, Yang X, Chen Z, et al. m(6)A-induced lncRNA RP11 triggers the dissemination of colorectal cancer cells via upregulation of Zeb1. Mol Cancer. 2019;18(1):87. doi:10.1186/s12943-019-1014-2
25. Yang M-H, Zhao L, Wang L, et al. Nuclear lncRNA HOXD-AS1 suppresses colorectal carcinoma growth and metastasis via inhibiting HOXD3-induced integrin $\beta 3$ transcriptional activating and MAPK/ AKT signalling. Mol Cancer. 2019;18(1):31. doi:10.1186/s12943019-0955-9

26. Liu T, Han Z, Li H, et al. LncRNA DLEU1 contributes to colorectal cancer progression via activation of KPNA3. Mol Cancer. 2018;17 (1):118. doi:10.1186/s12943-018-0873-2

27. Wang X, Yu H, Sun W, et al. The long non-coding RNA CYTOR drives colorectal cancer progression by interacting with NCL and Sam68. Mol Cancer. 2018;17(1):110. doi:10.1186/s12943-018-0860-7

28. Zhang R, Li J, Yan X, et al. Long noncoding RNA MLK7AS1 promotes proliferation in human colorectal cancer via downregulation of p21 expression. Mol Med Rep. 2019;19(2):1210-1221.

29. Li D, Jiang X, Zhang $X$, et al. Long noncoding RNA FGD5-AS1 promotes colorectal cancer cell proliferation, migration, and invasion through upregulating CDCA7 via sponging miR-302e. In Vitro Cellular \& Developmental Biology - Animal. 2019;55(8):577-585. doi:10.1007/s11626-019-00376-x

30. Xu R, Cao XR, Zhang BQ, et al. BLACAT1 is negatively associated with prognosis in patients with NSCLC and inhibits cell progression, metastasis and epithelial-mesenchymal transition through down-regulating Wnt/beta-catenin signaling pathway. Eur Rev Med Pharmacol Sci. 2019;23(14):6217-6225.

31. Bu C, Wang $\mathrm{B}$, Zhang $\mathrm{D}$, et al. Overexpression of the long non-coding RNA BLACAT1 promotes cell proliferation and invasion in colorectal cancer. Transl Cancer Res. 2019;8(1):35-43. doi: $10.21037 /$ tcr.2018.12.26

32. Huang FX, Chen HJ, Zheng FX, et al. LncRNA BLACAT1 is involved in chemoresistance of nonsmall cell lung cancer cells by regulating autophagy. Int J Oncol. 2019;54(1):339-347.

33. Ye X, Lv H. MicroRNA-519d-3p inhibits cell proliferation and migration by targeting TROAP in colorectal cancer. Biomed Pharmacother. 2018;105:879-886. doi:10.1016/j.biopha.2018.04.114

34. Huang R, Lin JY, Chi YJ. MiR-519d reduces the 5-fluorouracil resistance in colorectal cancer cells by down-regulating the expression of CCND1. Eur Rev Med Pharmacol Sci. 2018;22 (9):2869-2875.

35. Evert J, Pathak S, Sun XF, et al. A Study on Effect of Oxaliplatin in MicroRNA Expression in Human Colon Cancer. J Cancer. 2018;9 (11):2046-2053.

36. Dong Z, LncRNA WY. BLACAT1 accelerates the proliferation and migration of osteosarcoma cells through regulating STAT3. Pathol Res Pract. 2019;215(3):571-579.

37. Ye Q, Su L, Chen D, et al. Astragaloside IV Induced miR-134 Expression Reduces EMT and Increases Chemotherapeutic Sensitivity by Suppressing CREB1 Signaling in Colorectal Cancer Cell Line SW-480. Cell Physiol Biochem. 2017;43(4):1617-1626.

38. Bian Z, Jin L, Zhang J, et al. LncRNA-UCA1 enhances cell proliferation and 5-fluorouracil resistance in colorectal cancer by inhibiting miR-204-5p. Sci Rep. 2016;6:23892.

39. Yan L, You WQ, Sheng NQ, et al. A CREB1/miR-433 reciprocal feedback loop modulates proliferation and metastasis in colorectal cancer. Aging. 2018;10(12):3774-3793.

\section{Publish your work in this journal}

Cancer Management and Research is an international, peer-reviewed open access journal focusing on cancer research and the optimal use of preventative and integrated treatment interventions to achieve improved outcomes, enhanced survival and quality of life for the cancer patient.
The manuscript management system is completely online and includes a very quick and fair peer-review system, which is all easy to use. Visit http://www.dovepress.com/testimonials.php to read real quotes from published authors. 